\title{
Contributions à l'étude des Phasiinae cimicophages (Diptera Tachinidae)
}

\author{
38 - Inféodation à la plante nourricière de l'hôte \\ chez Phania pseudofunesta (Villen.) et Dionaea aurifrons \\ (Mg.) avec des remarques nomenclatoriales et taxinomiques
}

\author{
par Claude DUPUIS
}

Muséum national d'Histoire naturelle, 57, rue Cuvier, F 75005 Paris

Station de Parasitologie expérimentale et comparée, F 37120 Richelieu

\section{Résumé analytique}

Une liaison écologique constante entre un Diptère Phasiinae parasite, son Hétéroptère hôte et la plante nourricière de celui-ci avait été démontrée précédemment dans le cas de Gymnosoma intermedia. Ce type de liaison est illustré ici par les cas nouveaux de deux espèces dont les hôtes demeuraient encore inconnus: Phania pseudofunesta, parasite de Legnotus limbosus sur les Galium et Dionaea aurifrons, parasite de Dicranocephalus agilis sur les Euphorbia. L'auteur insiste sur les méthodes, tant d'observation sur le terrain que d'expérimentation, qui l'ont conduit à ces résultats à la Station de Parasitologie de Richelieu. Pour fixer correctement le statut taxinomique et nomenclatorial des deux Phasiinae, il est nécessaire d'introduire les novations suivantes: Weberia (R.D., non auct.) digramma (Mg.) nov. comb. = Lepidosyntoma digramma nov. syn.; Phaniaba nom. gener. nov. pro «Phania» vittata Meig., sp. typica ; Dionaea aurulans R.D., sp. rediviva distincta $=D$. flavisquamis R.D., nov. syn. 


\section{Synopsis}

Contributions to the study of the cimicophagous Phasiinae (Diptera Tachinidae) - 38 - Dependency of the host's foodplant in Phania pseudofunesta (Villen.) and Dionaea aurifrons (Mg.), with remarks on their taxonomy and nomenclature.

A constant ecological linkage between a parasitic Diptera Phasiinae, his host of the suborder Heteroptera and the foodplant of this host has been formerly demonstrated in the case of Gymnosoma intermedia. This type of linkage is exemplified in the present work by the new cases of two species, the hosts of which have hitherto remained unknown: Phania pseudofunesta, parasite of Legnotus limbosus living on Galium spp., and Dionaea aurifrons, parasite of Dicranocephalus agilis living on Euphorbia spp. The author emphasizes the methods of field observation as well as experimentation which allowed him to obtain these results at the parasitological Station of Richelieu (Indre \& Loire, France). For the correctness of the taxonomic status and nomenclature of the two Phasiinae, it is necessary to introduce the following changes: Weberia (R.D., non auct.) digramma (Mg.) nov. comb. = Lepidosyntoma digramma nov. syn.; Phaniaba nom. gener. nov. pro «Phania» vittata Mg., sp. typica ; Dionaea aurulans R.D., sp. rediviva distincta $=D$. flavisquamis R.D., nov. syn.

Plusieurs années d'observations suivies sur Gymnosoma intermedia Loew m'ont permis d'établir que ce Phasiinae était écologiquement lié à Stachys sylvatica, Labiée nourricière de son hôte, l'Hémiptère Pentatomide Eusarcoris fabricii Kirk. (Dupuis 1970, 1972).

Suspectant qu'une telle inféodation à la plante nourricière de l'hôte pouvait se retrouver chez d'autres Phasiinae également monophages, je me suis livré à quelques observations et expériences fort simples, mais suivies, qui me valent effectivement de faire connaître aujourd'hui deux espèces dans le même cas.

Bien que les Diptères considérés appartiennent à des tribus différentes de Phasiinae, leur étude dans une même publication illustrera la portée des méthodes qui, partant des mouches adultes, m'ont conduit à la découverte des hôtes, jusqu'alors inconnus de leurs larves.

Sous les rapports taxinomique et nomenclatorial, les deux espèces en cause exigent, comme cela est encore fréquent chez les Tachinaires, un certain nombre de précisions que j'apporterai in fine.

A M. le Professeur Henri Galliard, qui fut longtemps directeur de la Station de Parasitologie de Richelieu (Indre-et-Loire), il m'est agréable de dédier, pour son $80^{\circ}$ anniversaire, le présent travail qui repose avant tout sur des observations effectuées à Richelieu. 


\section{Biologie imaginale et hôte de Pbania pseudofunesta (Villen.)}

\section{A. - Introduction.}

Phania pseudofunesta (Villen.) («Weberia» curvicauda ou pseudofunesta des auteurs, v. infra III) est un Phaniina fort commun, dont on ignorait toutefois l'hôte véritable (1).

Depuis mon élevage de Phania (s. str.) incrassata Pandellé, parasite du Cydnide Sehirus bicolor (L.) (Dupuis 1963 : 103) et parce que la dissection d'autres représentants de cette famille, notamment Sehirus luctuosus M. \& R. (2) et Legnotus limbosus (Geoffr.), m'a livré de nombreuses larves « phanioïdes », je tiens pour assuré que toutes les Phania sont cimicophages, comme tous les Phasiinae. Il reste à préciser l'hôte de chacune.

J'ai déjà indiqué rapidement (Dupuis 1970: 1700) comment j'ai pu reconnaître dans Legnotus limbosus (Geoffr.) l'hôte de Phania pseudofunesta.

J'estime utile de détailler ici ce premier résultat et de le compléter par les observations écologiques qui démontrent l'existence d'un nouveau triplet « plante nourricière de l'hôte/hôte/parasite».

C'est l'observation du comportement imaginal non parasitaire du Phasiinae qui m'a mis sur la voie de la découverte de l'hôte. A Richelieu, de juillet à septembre, les $\delta^{A} \sigma^{A}$ de $P h$. pseudofunesta stationnent souvent sur les corolles de Calystegia sepium (= Convolvulus sp. in Dupuis 1970), d'où ils se précipitent sur tout insecte ou leurre qui passe à proximité. Ce comportement d'affût sexuel (Dupuis 1970), particulièrement fréquent en Station 48 (jeune peupleraie), y implique une concentration du Phasiinae, et par conséquent de son hôte. Le Calystegia n'hébergeant en propre aucun Hétéroptère spécifique, il fallait rechercher la cause de cette concentration parmi les plantes compagnes. J'ai vite songé à Galium aparine, dominant en Station 48 et cité comme plante nourricière de Legnotus limbosus, lui-même hôte (v. supra) de larves phaniö̈. des indéterminées.

L'hypothèse ainsi formée d'un triplet Galium/Legnotus/Phania a été vérifiée en deux temps: quant aux rapports parasitaires Legnotus/Phania d'abord, quant aux rapports écologiques entre les trois membres ensuite.

(1) De nombreux ouvrages l'indiquent comme parasite de Coléoptères Carabiques. L'on sait (cf. Herting 1957: 34, 1960: 146; Dupuis 1963: 64) que cette assertion repose sur l'observation réelle (Dalman 1828: 51) d'un parasite classique des Carabiques, Freraea gagatea R. D. 1830 (= Gymnopeza albipennis Zett. 1840), mais sur sa détermination erronée (Boheman 1829: 165) à une époque où ce Diptère n'avait pas encore été décrit. Je connais deux autres mentions erronées d'hôtes de Phania: celle que j'ai déjà dénoncée (1963: 65) d'une «Weberia sp. » élevée (?) du Noctuide Rhyacia pronuba (L.) (Séguy 1953: 92) et celle d'une «Weberia thoracica» parasite, selon Kiselev (1951: 99) du Tortricide Carpocapsu pomonella L. Dans ce dernier cas, il s'agit explicitement d'une «redétermination» (quand? par qui ?) de Thryptocera (Gymnopareia) pomonellae Schnabl \& Mokrzecki 1903 (cf. Mokrzecki 1903).

(2) A Richelieu, Phania (s. str.) speculifrons (Villen. 1919) nov. comb. et Sehirus luctuosus évoluent en nombre, en juin, sur les Myosotis de certaines stations. Il conviendra de vérifier si la larve phanioïde qu'héberge fréquemment ce Cydnide assez volumineux n'est pas précisément celle de cette Phania de grande taille, encore mal connue (cf. Dupuis 1965: 92-93). 


\section{B. - Vérification des rapports parasitaires Legnotus/Phania.}

\section{1) Les MÉthodes possibles de VÉRIFICATION.}

Pour établir qu'une espèce de Phasiinae (ou de Tachinaire, ou d'Insecte entomophage), dont on ignore tout quant à l'hôte, est parasite d'un Hétéroptère (ou d'un Insecte) donné, l'on dispose de deux méthodes :

- ou l'élevage du parasite adulte à partir d'un hôte naturellement infesté (méthode générale) ;

- ou l'obtention in vitro de la ponte et du développement du parasite dans un hôte neuf (méthode à utiliser avec précaution dans le cas des polyphages ou des insectes à spécificité xénique plus écologique qu'éthologique).

Les espèces pour lesquelles on dispose de matériaux larvaires de comparaison (polyphages déjà élevées de certains hôtes ; mono/oligophages élevées en partie $o b$ ovo ou ex pupa) sont passibles d'une troisième méthode, qui tient un peu de chacune des précédentes :

- la détermination des larves parasites trouvées à la dissection d'hôtes infestés naturellement.

Dans le cas de Ph. pseudofunesta, vu la difficulté de capturer en temps opportun un grand nombre de Legnotus limbosus, je n'ai pas encore pu utiliser la première méthode. La deuxième m'a fourni les résultats concluants que j'expose ci-dessous. La troisième, en l'espèce l'identification à $P h$. pseudofunesta de larves I et III issues de dissections anciennes de Legnotus, ne m'a été accessible que tout récemment. Il m'a fallu attendre de disposer de matériaux de comparaison de larves I (obtenues expérimentalement, v. infra) et d'armatures buccales de larves III (extraites de puparia pris dans le sol et ayant donné des imago, v. infra). Cette identification confirme pleinement que Legnotus limbosus est naturellement l'hôte de Phania pseudofunesta.

\section{2) VÉRIFICATION EXPÉRIMENTALE.}

Pour assuré que soit le résultat qui précède, l'intérêt de mes expériences d'infestation in vitro de Legnotus par Ph. pseudofunesta demeure entier. En effet, d'une part très peu d'infestations par Phasiinae à ponte dans l'hôte ont été obtenues au laboratoire (Dupuis, 1963 : 238-240), d'autre part, le protocole expérimental et les précautions prises peuvent s'appliquer à tous les Phasiinae encore trop nombreux dont on ignore les hôtes.

\section{a) Protocole expérimental.}

Les expériences, fort simples, consistent à mettre en présence d'une $q$ de Phania pseudofunesta de la nature un Legnotus limbosus, imago ou larve, indemme (hôte «neuf ») et, si la ponte est obtenue, à suivre le développement des œufs.

Chez tous les Phasiinae, l'accouplement se produit quelques heures après l'émergence imaginale, de sorte que les $\$ \uparrow+$ récoltées dans la nature sont en très grande majorité fécon- 
dées et (à l'inverse des $\nmid \uparrow$ vierges) capables de pondre (cf. Dupuis 1963: 184, 205). Les 우 de $\mathrm{Ph}$. pseudofunesta récoltées dans la nature et que j'ai utilisées ici n’ont pas fait exception à cette règle.

Dans le cas d'un hôte, tel Legnotus limbosus, que l'on ne sait pas élever, les hôtes expérimentaux proviennent de la nature. On écartera le risque d'expériences inutiles avec des sujets déjà parasités en observant un délai de quarantaine. La dissection - qui permet toujours de faire le départ entre parasites anciens éventuels et parasites nouveaux dira si l'hôte était réellement neuf, ce qui fût le cas dans mes expériences.

Le récipient de contention des partenaires est un couvercle de tube Borrel renversé, pourvu d'un fond de carton ondulé et fermé par un verre de montre (op. cit., p. 20).

Les résultats sont établis par la dissection des hôtes.

$\mathrm{Si}$ cette dissection suit immédiatement l'expérience, on obtient des œufs dont il importe de savoir s'ils sont susceptibles de développement. Phania pseudofunesta appartient à une tribu de Phasiinae qui pondent dans le corps de l'hôte; ses œufs sont néanmoins capables de se développer et d'éclore hors de celui-ci. On les laisse dans le verre de montre ayant servi à la dissection, en enlevant tous les débris organiques et la presque totalité de l'eau; le tout est placé en chambre humide (boîte de Pétri fermée, à fond pourvu d'un papier filtre entretenu mouillé).

Si la dissection est différée de quelques jours par rapport à l'expérience, on pourra suivre le développement des æufs, puis des larves, dans l'hôte. Je n'ai pas disposé d'assez de Legnotus pour réaliser un nombre d'expériences qui m'ait permis de suivre plus que le début de ce développement.

\section{b) Résultats.}

Exp. I - Hôte : L. limbosus, stade V $q$, récolté le 10-viII-1968, en Station autre que 48 (probabilité accrue de matériel neuf) en battant des Galium aparine en fruits accrochés à des basses branches de pin. - Quarantaine jusqu'à l'expérience. - Parasite: Ph. pseudofunesta, \& IP 508, récoltée le 14-vIII-1968 en Station 48. - Contact du 14-VIII, 15 h environ au 15-VIII, 23 h 30. - Dissection aussitôt. - L'hôte renferme, dans la cavité générale, 23 œufs parasites et présente une douzaine de minimes traces traumatiques à la face inférieure du thorax et de l'urite II. - Sur 11 œufs transférés en chambre humide, 9 ont éclos le $17-\mathrm{vIII}$ de 11 à $20 \mathrm{~h}$.

Exp. 11. - Hôte: L. limbosus, stade $\mathrm{V}$ ở proche de sa mue imaginale; mêmes provenance et quarantaine. - Parasite: même ? IP 508. - Contact du 15-viII, 23 h 30 au 16-VIII, 22 h 30. - Dissection aussitôt. - L'hôte renferme 2 æufs qui éclosent en chambre humide le 20-vIII, $10 \mathrm{~h}$.

Exp. III - Hôte: L. limbosus, jeune imago $\delta^{*}$, issu de stade $\mathrm{V}$ après capture ; mêmes provenance et quarantaine. - Parasite: même $q$ IP 508. - Contact du 17-vIII, 21 h 45 au 19-VIII après-midi. - Dissection différée jusqu'au 29-vIII. - L'hôte renferme 13 larves I de Phania: 1 vivante et 12 mortes.

Exp. IV - Hôte: L. limbosus, stade $\mathrm{V} \delta^{\hat{\sigma}}$; mêmes provenance et quarantaine. Parasite: Ph. pseudofunesta, \& IP 509, 16-VIII-1968, Station 48. - Contact du 16-vill, 17 h 15 au 18-vIII, 9 h. - Dissection aussitôt. — L'hôte renferme 17 œufs, la plupart dans le thorax, mais se libérant dans l'eau à la dissection. - 10 œufs transférés en chambre humide ont éclos le 20-vIII, de 10 à 19 h. 
Exp. V - Hôte: L. limbosus, imago + , récolté à ce stade en Station 48, le 8-IV-72, alors que les larves hivernantes de Phania ont déjà abandonné les hôtes (v. infra), mais néanmoins gardé en quarantaine jusqu'à l'expérience. - Parasite: Ph. pseudofunesta, † IP 751, 5-v-1972, Station 48. - Contact du 5-v, 21 h environ au 8-v, 16 h environ (mort de la mouche). — Dissection aussitôt. — L'hôte renferme 1 œuf parasite.

\section{c) Discussion.}

Il ne fait aucun doute que les œufs obtenus aient été pondus par les $q$ q utilisées. En effet :

$1^{\circ}$ et $2^{\circ}$ il n'y a guère de probabilité d'observer dans la nature, chez un seul hôte, des surinfestations comparables à celles des expériences $I, I I I$ et $I V$ ni des œufs présentant un développement à ce point synchrone;

$3^{\circ}$ l'éclosion en chambre humide dans les 3-4 jours suivant l'expérience de ponte correspond à une durée d'incubation courante chez les Phasiinae (cf. Dupuis 1963: 248251, 265-266) et inférieure à la durée de quarantaine des hôtes utilisés ;

$4^{\circ}$ les petites pontes ont été obtenues sur hôte proche de la mue $(\operatorname{Exp} . I I)$ ou de la part d'une $q$ affaiblie (Exp. V), circonstances dont je possède $n$ cas inédits chez divers Phasiinae.

Il est également certain que Legnotus limbosus convient au développement des œufs et des larves (cf. 13 éclosions sur 13 œufs dans l'expśrience III où la survie d'une seule larve résulte d'une élimination régulière chez les parasites solitaires que sont les Phasiinae).

Mes expériences indiquent donc clairement que Legnotus limbosus est un hôte convenable, tant aux $q 9$ qu'aux larves de $P h$. pseudofunesta.

d) Appendice: l'auf de Phania pseudofunesta.

Comme chez tous les Phasiinae à ponte dans l'hôte, l'œuf de Ph. pseudofunesta est fusiforme à chorion mince (mais élastique), avec un pôle arrondi et l'autre effilé. Mesuré dans l'eau dès son extraction de l'hôte, il présente une longueur de 500-550 $\mu$ et un diamètre de 125-150 $\mu$. L'œuf ovarien, à un stade non précisé de la choriogénèse, est plus gros (Dupuis 1963: 42). Lors de chocs asphyxiques de ㅇํ de Ph. pseudofunesta par l'acétate d'éthyle, j'ai pu constater qu'au moment de sa sortie du vagin exertile, l'œuf mou est très filiforme; il se renfle aussitôt après en massue par l'un des pôles et tout le reste de l'œuf prend rapidement le même diamètre. Un œuf ainsi obtenu et transporté dans l'eau, conserve un chorion mou et des dimensions supérieures à celles de l'œuf extrait de l'hôte. Je conclus de ces observations que les dimensions «vraies» sont celles que l'œuf acquiert dans l'hôte. Quant à la plasticité de l'œuf à la ponte - phénomène très général chez les Phasiinae - elle équivaut, ici et ailleurs (par exemple chez le Cylindromyiina), à une «adaptation » à l'injection dans l'hôte par une perforation de petit diamètre.

On notera qu'en chambre humide, l'armature bucco-pharyngienne de la larve I apparaît au pôle arrondi de l'œuf qui, conformément à mes remarques précédentes (op. cit., p. 43), représente donc bien le pôle antérieur.

\section{C. - Vérification des rapports écologiques Galium/Legnotus/Phania.}

La connaissance de l'hôte de $P h$. pseudofunesta, acquise grâce à l'hypothèse d'un triplet plante/punaise/mouche, ne suffit cependant pas à prouver la réalité écologique 
permanente de ce triplet. Il importait de vérifier, ce que j'expose ci-après, que 1) L. limbosus existe, au cours des saisons et des années, dans les stations à Galium et Phania, 2) Galium aparine offre au Cydnide, et celui-ci à Ph. pseudofunesta, la possibilité de cycles annuels complets successifs, 3) les stations à Galium permettent, et surtout favorisent, les comportements non parasitaires du Diptère.

\section{1) CoÏnCidence stationnelle.}

A Richelieu, Phania pseudofunesta est très commune en toutes sortes de stations mésophiles telles que jeunes peupleraies (Stations 48, 3 bis) et chemins forestiers (Station 60), mais sensiblement moins abondante dans les stations ensoleillées et sèches (friches et coupes sableuses). Ceci correspond, en première approximation, à l'écologie même de Galium aparine, espèce mésophile de la zone tempérée, originaire des milieux boisés frais et largement répandue dans les peupleraies-aulnaies anthropophiles des vallées (Gérard Aymonin in litt.).

Legnotus limbosus existe bien dans ces mêmes stations à Galium, où je l'ai noté dès 1970 (en juin). Selon toute probabilité, il y hiverne dans le sol, au pied de la plante, et c'est là même que les parasites ayant hiverné dans l'hôte abandonnent celui-ci. En effet, les 8 et 9 avril 1972, j'ai trouvé en grattant le sable au pied des Galium de la Station 48, non seulement 8 imagos du Cydnide (indemnes de parasites), mais aussi, à quelques centimètres, deux petits puparia d'où sortirent, le 17 avril, un $\delta$ et une $q$ de Phania pseudofunesta.

L'hivernage de l'hôte et du parasite dans la station où se poursuit leur développement vernal et estival explique la pérennité du peuplement en un même lieu et pourquoi, en Station 48 par exemple, j'observe $P h$. pseudofunesta depuis 1953 !

\section{2) COÏNCIDENCE PHÉNOLOGIQUE.}

L'on connaît assez mal les biologies du Galium et du Legnotus, notamment l'évolution de leurs relations saisonnières. De la Phania, dont j'ai surtout observé la phénologie imaginale, j'ignore encore le rythme de développement larvaire. Malgré ces lacunes, je puis apporter sur la coïncidence phénologique de la plante, du Cydnide et du Phasiinae, les solides données préliminaires suivantes.

a) Galium aparine.

A Richelieu, dans la jeune peupleraie de la Station 48, G. aparine forme dès la fin d'avril de longues tiges, rampantes, puis dressées jusqu'à $80 \mathrm{~cm}$ en mai où il couvre alors la majeure partie du terrain.

En juin, ses longues tiges traçantes, accrochées dans la végétation, portent des fleurs à l'apex et des fruits à des degrés croissants de maturité de l'apex à la base. La plante est toujours bien visible et accessible. En juillet-août, elle est dominée par une végétation envahissante de Rubus, Heracleum, Symphytum, Epilobium, Cirsium, Urtica, Stachys, Calystegia, etc... 
Elle peut être desséchée et en fruits, dès la mi-juillet en certains stations, mais non toutes. On trouve ses fruits au sol, de l'été au printemps.

Ce cycle, dans ses grandes lignes, est parfaitement compatible avec ceux que j'examine ci-après, de la punaise et de la mouche. Une meilleure connaissance des exigences du Legnotus et des comportements de Ph. pseudofunesta imposeront cependant une étude plus approfondie de la biologie du Galium, notamment quant à la fructification.

\section{b) Legnotus limbosus.}

La biologie de L. limbosus n'est connue que par des indications très fragmentaires (une date, une plante...) éparpillées dans les travaux de divers observateurs, par exemple Schumacher (1916 : 212), Butler (1923 : 36-37), Gulde (1921 : 339), Michalk (1938 : 48-49), Korinek (1940: 237-239), Jordan (1940: 101), Singer (1952: 2), Cobben (1953 : 172), Thomas (1954: 26-28), Halaszfy (1954: 106-107), Southwood \& Leston (1959: 24), Putchkov (1961 : 89-90), Wagner (1966 : 31). D'après ces indications et quelques données personnelles, je pense, néanmoins, pouvoir en exprimer comme suit les traits essentiels.

Legnotus limbosus est univoltin et hiverne au stade adulte. Les imagos d'ancienne génération (i.e. ayant passé l'hiver) subsistent tout le mois de mai et une grande partie de juin. La copulation et la ponte ont lieu durant ces mêmes mois. Les larves s'observent en juin-juillet et jusqu'au milieu d'août sur Galium (CD !), mais non en tous lieux ni à toutes heures et ceci reste à éclaircir. Les imagos de nouvelle génération apparaissent dès août.

L'espèce vit aux dépens des Galium aparine, G. verum, G. mollugo, et sur les Galium en général. Sa capture sur d'autres végétaux, y compris les Labiées, correspond peut-être à un erratisme imaginal à la fin de l'hivernage et au moment des copulations ou encore à la diaspora des larves II au terme des soins maternels aux œufs et larves néonates, soins qui sont donnés dans le sol.

La signification des diverses parties des Galium pour l'alimentation de la punaise reste à préciser. J'ai noté que $G$. aparine est en fruits au moment du développement des larves.

J'ignore si les jeunes imagos de nouvelle génération doivent nécessairement continuer de se nourrir ; leur présence en août et septembre au pied des Verbascum thapsiforme $(\mathrm{CD}$ !) pourrait correspondre à une estivation qui se continuerait directement par l'hivernage (il existe ainsi, chez un autre Cydnide, Cydnus $[=$ Brachypelta $]$ aterrimus [Först] une diapause imaginale de 7-8 mois, fide Schorr 1957 : 563). Cette estivation prendrait place au moment où les Galium commencent à se dessécher.

Toutes les espèces de Cydnides sont, dans une large mesure, terricoles et fouisseuses ; toutefois, d'après mes observations de Sehirus bicolor (L.) sur les Labiées, de S. luctuosus M. R. sur les Myosotis ou de Cydnus aterrimus sur les Euphorbia, nombre d'entre elles présentent des phases de vie aérienne. 
L. limbosus est dans ce cas; j'ai rapporté ci-dessus la récolte de ses larves par battage de Galium aparine accrochés à des basses branches de pin ; Michalk (1938: 43) indique qu'on peut le récolter au filet fauchoir (gestreifen) au moment des copulations.

Quoi qu'il en soit de leur périodicité (saisonnière ou nycthémérale) et de leur déterminisme (exogène ou physiologique), ces phases épigées ont une évidente signification parasitologique. Elles permettent de comprendre pourquoi les Phasiinae parasites de Cydnides (Heliozeta pellucens [Fall.], Gymnosoma brachypeltae Dup., Phania spp.) sont morphologiquement tout aussi peu «fouisseurs» que leurs congénères ou alliés parasites de Pentatomoidea « aériens».

\section{c) Phania pseudofunesta.}

L'histogramme des fréquences imaginales par décades de $P$ h. pseudofunesta (600 imagos récoltés) présente deux très nets maxima annuels, ce qui implique deux générations certaines.

Le premier maximum dans la $2^{\circ}$ décade de mai correspond à l'apparition, d'emblée massive, des imagos de $1^{\text {re }}$ génération. Ce synchronisme indique, là comme ailleurs (Dupuis 1968: 31, 33), que tous les individus hivernent au même stade dans des hôtes tous imagos et que les conditions extérieures seules règlent à la fois l'abandon des hôtes (le 11 avril 1948, un cas de St-Germain-en-Laye, Yvelines; avant le 8 avril 1972 à Richelieu), la pupaison et l'émergence imaginale (les imaginalisations obtenues le 17 avril — v. supra — ont été accélérées par les conditions du laboratoire). L'abondance de cette génération qui coïncide avec la poussée vernale du Galium décroît très vite, les mouches ne s'observant guère que 5 semaines; les Legnotus dont elles peuvent alors disposer pour pondre sont toujours des imagos d'ancienne génération (un Legnotus de St-Germain-en-Laye, le 23 mai 1950, hôte d'une larve I).

Le second maximum, dans la $2^{\circ}$ décade de juillet, est précédé et suivi, du début de juillet au milieu d'août, par une longue période de grande abondance des Phania adultes. Cet étalement de la $2^{e}$ génération reflète, à n'en pas douter, l'échelonnement des pontes des imagos de la $1^{\text {re }}$. Les Phania de $2^{\circ}$ génération disposent essentiellement, pour pondre en juillet-août, des larves (sur Galium, CD !), ou, aux mieux, des jeunes imagos de Legnotus (Michalk [1938: 48-49], signale une larve de Diptère indéterminée chez des «noch frisch entwickelte, unausgefärbte Stücke» pris en août).

La manière dont se poursuit le cycle reste à établir. Les Phania adultes, observées en assez faible abondance de la mi-août à la première décade d'octobre, permettent de croire à une troisième génération partielle. Le cas serait alors semblable à celui de Gymnosoma intermedia (cf. Dupuis 1972): les œufs pondus par les +9 de $2^{\circ}$ génération dans les larves les plus âgées ou les nouveaux imagos les plus précoces de l'hôte donneraient des mouches adultes de $3^{e}$ génération, mais l'effectif principal, pondu dans de jeunes larves, donnerait la génération hivernante (première de l'année suivante).

Quelque précision qu'apportent, à ce sujet, les observations à venir, le fait que Phania pseudofunesta, espèce bivoltine, ait pour hôte un Cydnide univoltin, n'entrave 
en rien la réalisation du cycle, la mouche pouvant pondre indifféremment sur les larves et imagos de son hôte (v. expériences ci-dessus).

La permanence du système Galium-Legnotus-Phania est donc phénologiquement possible. Il restera à établir le rôle éventuel du Galium comme facteur favorisant la découverte de l'hôte.

\section{3) Comportements NON PARAsitaires de Phania pseudofunesta Dans Les stations A Galium.}

L'alimentation et le repos des Phasiinae adultes ne dépendant aucunement d'un microcosme végétal déterminé, il n'y a pas lieu de détailler ici comment les stations à Galium offrent, en toutes saisons, à Phania pseudofunesta, des fleurs à butiner et des supports érigés de repos nocturne. On notera simplement qu'en mai 1972, en Station 48 , les tiges de Galium aparine étaient le support de repos vespéral de loin le plus fréquenté par ce Diptère (...mais aussi — ce qui écarte toute idée de spécificité — le végétal dressé de beaucoup le plus abondant).

L'accouplement de la mouche, par contre, semble bien favorisé par le Galium.

Il existe, chez Ph. pseudofunesta comme chez divers autres Phasiinae (Dupuis 1970), trois types possibles de rencontre des sexes : la rencontre fortuite sur les fleurs d'alimentation, l'affût sexuel des $\delta \delta^{\wedge}$ et enfin la recherche active par ceux-ci du paztenaire sexuel. Les deux derniers de ces comportements dépendent, dans une large mesuie, de la plante nourricière de l'hôte.

Quant à l'affût sexuel de $P h$. pseudofunesta, j'avais déjà noté (op. cit.) le guet des $\delta^{0} \sigma^{\circ}$ de deuxième génération sur les corolles de Calystegia mêlées au Galium en été ;

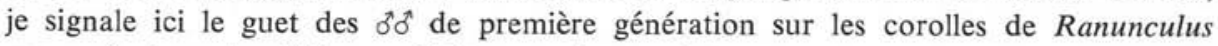
repens également mêlées au Galium, mais au printemps.

Quant à la recherche active du partenaire sexuel, que je ne connaissais pas encore dans cette espèce, mes observations sont les suivantes.

Des $\delta^{A} \delta$ ont été vus, d'une part le 7 mai 1972, explorant sommairement des touffes de Galium, tout comme les $\delta \delta^{\top}$ de Gymnosoma intermedia explorent des touffes de Stachys sylvatica, d'autre part, le 8 mai, se poursuivant, rejoignant et séparant, dans des touffes de Myosotis mêlés au Galium.

Ces observations, encore incomplètes, sont néanmoins satisfaisantes car elles montrent, chez des Phasiinae distincts, des comportements comparables dans lesquels la plante nourricière de l'hôte joue le même rôle, sinon de «signal», du moins de «cadre » favorisant ces comportements.

En conclusion de ces observations sur la coïncidence stationnelle et phénologique Galium-Legnotus-Phania et sur le Galium en tant qu'élément cadre du microcosme de la mouche, je considère la liaison de la plante, de la punaise et de son parasite comme suffisamment étroite pour servir de guide très sûr à la collecte d'observations complémentaires sur un Diptère dont toute la biologie était inconnue.

Je tiens à rappeler, pour souligner la nature si particulière du travail de terrain, que cette conclusion méthodologique repose sur l'analyse de plus de 600 captures de 
Phania pseudofunesta depuis une vingtaine d'années et sur la lumière décisive apportée par quatre années d'observations suivies sur une espèce de biologie similaire (Gymnosoma intermedia).

\section{II. - Biologie imaginale et hôte de Dionaea aurifrons (Mg.)}

Dionaea aurifrons (Meig.) est un Leucostomatina plus ou moins rare, et de ce fait mal connu (cf. en III ci-après sa distinction d'avec D. aurulans). C'est, là encore, l'étude de la biologie imaginale qui m'a permis la découverte de l'hôte jusqu'alors ignoré.

Jusqu'en juillet 1970, je n'avais que très occasionnellement récolté l'espèce. Toutefois, il ne m'échappa pas, dès que je disposai de quelques individus pris à Richelieu lors de chasses de routine, que 6 d'entre eux (sur 9) avaient été capturés sur des Euphorbia seguieriana en fleurs et fruits dans la Station 36 (plantation localement clairsemée de jeunes pins sylvestres sur sol sablo-calcareux dénudé).

Je savais de longue date, par des dissections - également de routine - , que l'Hémiptère-Hétéroptère Dicranocephalus agilis (Scop.), inféodé aux Euphorbia, était parasité par des larves « dionaeoides» que je ne pouvais rapporter à aucune des rares larves de Leucostomatina déjà identifiées.

Pour les mêmes raisons que précédemment, je formai l'hypothèse de l'existence d'une relation entre Euphorbia, Dicranocephalus et Dionaea. Je procédai aussitôt, durant tout le mois d'août 1970, à des récoltes systématiques de Dicranocephalus et de Dionaea sur les Euphorbia seguieriana de la Station 36.

Les résultats, conformes à l'hypothèse de travail, furent:

- d'une part, l'obtention, le 14 août, d'un imago $\delta$ de Dionaea aurifrons à partir d'un puparium formé le 3 par une larve III «dionaeoïde» issue d'une $q$ de Dicranocephalus agilis (cas P 3578);

- d'autre part, la récolte sur l'Euphorbia de 24 individus de Dionaea aurifrons, espèce rare... dans les collections du moins.

A ces matériaux s'ajoutent, évidemment, des larves des trois stades de la Dionaea, extraites par dissection des Dicranocephalus et déterminées par comparaison avec les exuvies larvaires de stade III (dans le puparium), III et I (dans l'hôte) de l'imago du cas P 3578.

Depuis, un sondage à Chaveignes (Indre-et-Loire), dans une friche à Euphorbio seguieriana (d'où Bernard Ehanno m'avait rapporté un Dicranocephalus parasité), m'a encore permis, le 28-vir-1971, la récolte, sur cette plante, de $2 \sigma^{\circ} \sigma^{\circ}$ de $D$. aurifrons.

$\mathrm{Au}$ total, 33 sur 36 de mes imagos de cette espèce ont été pris sur la plante nourricière de l'hôte, et les trois autres — comme il est banal s'agissant de Phasiinae sur des fleurs d'Ombellifères. Ces chiffres mêmes confirment la liaison écologique Euphorbia/Dicranocephalus/Dionaea aurifrons. 
Le cycle annuel de la mouche semble au moins bivoltin. Je soupçonne une première génération en juin-juillet (7 imagos sur 36 pris du 13-vI au 18-vII) (3). L'hôte vivrait alors surtout sur Euphorbia cyparissias (qui, à Richelieu comme ailleurs, cf. Schorr, 1957: 581, fleurit et fructifie avant E. seguieriana et dans les mêmes stations).

La deuxième génération de fin juillet à fin août (28 individus sur 36, du 28-vII au 30-viII) est celle que j'ai surtout observée ; l'hôte vit alors sur E. seguieriana.

Une troisième génération partielle, à l'automne, paraît possible (1 individu le 29-1X).

Quelque précision que doivent apporter sur ce cycle des observations plus nombreuses, la coïncidence phénologique avec l'hôte univoltin Dicranocephalus de la mouche plurivoltine $D$. aurifrons ne présente aucune difficulté de réalisation, ce Phasiinae pouvant attaquer, ainsi que me l'ont prouvé mes dissections, les stades préimaginaux de la punaise.

\section{Remarques taxinomiques et nomenclatoriales}

\section{A. - Les genres Phania Mg. et Weberia R.D.}

L'épithète pseudofunesta, Villeneuve, 1931: 70 est un nomen novum pour l'espèce curvicauda, Meigen, 1824: 221 et auct., non Fallén, 1820: 17. En raison de la priorité de sa désignation comme type du genre Phania, Meigen, 1824: 218, par Westwood, 1840: 140, cette espèce ne peut être combinée avec des noms de genres synonymes ultérieurs. Le genre Weberia R.D. datant de 1830, la combinaison «Weberia pseudofunesta», dont, avec bien d'autres, j'ai jusqu'alors largement usé, est donc formellement fautive.

Taxinomiquement, l'erreur est plus grave encore, car Weberia appendiculata, Robineau-Desvoidy, 1830: 233, espèce unique et type du genre, n'est ni Phania curvicauda auct. (contra Bezzi, 1907 : 564), ni une Phania (contra Macquart, 1835: 184). ni même un Phaniina!

Le type de Robineau-Desvoidy existe toujours dans les collections du Muséum à Paris (coll. R.D. $n^{\circ}$ 293). Le $D^{r}$ Benno Herting (Stuttgart) qui s'est, le premier, donné la peine de le débarrasser de ses moisissures et de l'examiner m'a écrit, le 23 avril 1970: "C'est une $q$ de Lepidosyntoma digramma Meigen! Je ne possède pas cette espèce, mais j'ai pu la reconnaître avec l'aide de votre description [i.e. Dupuis,

(3) Plus encore aujourd'hui qu'en 1963 (p. 97), je tiens pour probable que la «Dionaea forcipata » élevée par Michalk (1933: 129) d'un Dicranocephalus agilis récolté le 10 juin représente en réalité une $D$. aurifrons qui serait, en ce cas, de première génération. La détermination des Dionaea (v. nira) et Labidogyne (type forcipata Mg.) est restée, en effet, si délicate jusqu'à ce jour, qu'une idenification avancée il y a 40 ans, sans éléments diagnostiques ni garantie de taxinomistes, ne peut être icceptée d'emblée. Je confirme en outre n'avoir jamais trouvé chez Dicranocephalus les larves de la saie Labidogyne forcipata (Mg.) que j'ai élevée d'Enoplops scapha (F.) (loc. cit.). 
1968: 19-23]. L'abdomen était presque enroulé avant la préparation et la pince était dirigée en bas. »

Je confirme ici totalement cette détermination de mon estimé collègue. Malgré son mauvais état (la tête manque, les ailes sont frippées et l'abdomen réduit à peu de choses), le type de Robineau, grâce à ses génitalia opportunément épargnées par les anthrènes est bien reconnaissable comme une $q$ de Leucostomatina à pinces acroabdominales massives de Tachina digramma, Meigen, 1824: 346!

Le repliement de l'abdomen d'un spécimen desséché sans grand soin n'a rien de surprenant, dans une espèce où, chez la $q$, il est long, «traînant » et \& se meut... facilement de côté » (Dupuis, op. cit., p. 21) ; ce repliement entraînait une position anormale de la pince que Robineau (1830: 233) a interprétée comme «deux petits appendices sous le deuxième segment abdominal ».

Tout devient donc parfaitement clair et le nom générique de Weberia doit être substitué à celui de Lepidosyntoma pour la mouche en question, qui se nommera Weberia digramma (Meigen, 1824) nov. comb. $=W$. appendiculata R.D. nov. syn. (pour les autres synonymes, cf. Dupuis, 1968: 18) (4).

Quant à la pratique schinérienne d'un genre «Phania» restreint à $P h$. vittata, Meigen, elle est nomenclatorialement inacceptable, car vittata n'est pas le type de Phania. Townsend (1916: 12) a proposé comme nom de substitution Euphania, qui se trouve malencontreusement préemployé. Je propose ici, pour préserver, l’ordre alphabétique, le nom générique, délibérément barbare et féminin, de Phaniaba, nom. nov., espèce type : Phania vittata, Meigen, 1824: 219.

\section{B. - Dionaea aurifrons (Mg.) et D. aurulans R.D.}

Dionaea, Robineau-Desvoidy, 1830, s. str. (cf. Herting, 1966: 10) et Eulabidogaster, Belanovsky, 1951 (type setifacies, Rondani) se distinguent des autres genres paléarctiques de Leucostomatina à cellule alaire apicale ouverte (Clairvillia et affines, cf. Dupuis, 1968 + Labidogyne, type forcipata, Mg.) par une série de dents au bord inférieur des branches de la pince acroabdominale des $\subsetneq \uparrow$. C'est un caractère que l'on retrouve chez les Leucostoma (à cellule apicale pédonculée).

Les gènes sont ciliés chez Eulabidogaster et nus chez Dionaea.

Il existe en France deux espèces de ce dernier genre :

- l'espèce-type, Dionaea aurifrons (Meigen, 1824) (= lineata Rob.-Desv., selon la synonymie de Herting, 1966: 10 que confirme mon examen des spécimens types) ;

- une espèce méconnue, toute différente, Dionaea aurulans, Robineau-Desvoidy. 1830.

(4) En révisant le type $q$ de Tachina digramma - aujourd'hui Weberia digramma (Mg.) j'avais signalé (1968: 16, n. 14) qu'il est accompagné, dans la collection Meigen d'un $\delta$ de «Weberia * (sensu Bezzi et auct.): il faut lire un 3 de Priania! 
La bibliographie diptérologique n'apporte aucun moyen de les distinguer, car elles furent déjà confondues par Pandellé (1894: 78) et sont, unanimement et à tort, tenues pour synonymes depuis Villeneuve $(1901: 85 ; 1906: 247 ; 1907$ : 380) (5).

L'absence de Dionaea aurulans dans mon matériel personnel ne me permet pas une étude comparative fine et exhaustive de ces espèces dont je me contenterai ciaprès d'indiquer les caractères les plus évidents (à l'exclusion des caractères des genitalia des $\nmid \uparrow$ dont je n'ai pas assez de matériel pour une dissection).

On trouvera dans mes listes des matériaux étudiés les quelques synonymies certaines établies d'après les types; les synonymies additionnelles qui pourraient résulter de l'exégèse des auteurs ne seront pas examinées pour le moment.

\section{1) Dionaea aurifrons (Meigen).}

\section{a) Principaux caractères diagnostiques.}

Jị: Thorax, sur toute sa longueur, nettement rayé de cendré sur fond noir (d'où le nom synonyme de lineata R.D.) ; scutellum largement cendré ; 1 (\$) ou 1-2 ( $\left.\sigma^{*}\right)$ lignes de sétules orbitales en dehors des macrochètes frontales; orbites à pruinosité homogène très épaisse sous les sétules - $\sigma^{\lambda} \sigma^{*}$ : orbites dorées; sétules parafrontales brèves; soies des joues (en dehors des péristomales) courtes; vertex large comme la moitié de l'œil; orbite 1,5 fois plus large que la bande frontale; macrochètes marginales formant un rang complet à l'arrière des tergites abdominaux I-II, III, IV et $\mathrm{V}$, les médianes dans l'alignement des latérales; tergite I-II noir, les trois suivants saupoudrés d'une très épaisse pruinosité grise, évidente à l'œil nu; forceps médian en forme d'onglet triangulaire à pointe aiguë, canelée sur les côtés et comprimée latéralement à l'apex — $९$ : macrochètes abdominales formant, sur les tergites abdominaux I-II et III, un rang complet de marginales dont les médianes sont au même niveau que les latérales, et sur le tergite IV un rang complet inséré au quart postérieur de l'urite; tergite I-II noir, les deux suivants marqués dans leur moitié antérieure d'une pruinosité grise qui n'est épaisse que latéralement.

b) Matériaux examinés.

Types:

- holotype ơ de Tachina aurifrons Meigen, 1824 (Muséum, Paris, Collection Meigen, $\left.\mathrm{n}^{\circ} 1557\right)$;

(5) A défaut des types d'aurulans de Robineau (retrouvés longtemps après, cf. Royer, 1э31), Villeneuve a comparé le type $\delta$ d'aurifrons Meigen au matériel non typique et composite de Pandelié (v. infra). La mystique du recours aux «types», l'autorité de Villeneuve et 'enregistrement immédiat de sa «synonymie » dans le catalogue de Berzi (1907: 329) - qui omet d'ailleurs de citer ses sourzes - ont abusé tous les diptéristes subséquents. 
- holotype $q$ de Dionaea lineata Robineau-Desvoidy, 1863, nom. nov. pro Dionaea forcipata Robineau-Desvoidy, 1830 non Mg., 1824 (Mus. Par. Coll. RobineauDesvoidy, $\mathrm{n}^{\circ} 300$, sub. nom. «Dionaea forcipata $\mathrm{Mg}$. »);

- 2 allotypes ơ de Dionaea lineata Robineau-Desvoidy, 1863 (Mus. Par. Coll. Robineau-Desvoidy, $\mathrm{n}^{\circ}$ 301);

- 2 syntypes $\delta$ de Dionaea binotata Robineau-Desvoidy, 1863 (Mus. Par. Coll. Robineau-Desvoidy, $\mathrm{n}^{\circ}$ 303).

Matériaux de diverses collections:

$-1 \delta^{*}$ déterminé «Clairvillia aurulans in coll. Pandellé (Mus. Par., $\mathrm{n}^{\circ} 1823$, individu de Gavarnie ou Tarbes, à label rose; 1 autre $\delta$ et $1 q$ sont aurulans) ;

- 1 ơ déterminé \& Labidogaster aurifrons Meig. » (écriture de J. Villeneuve), presqu'île de Rhuys (Morbihan), J. Surcouf leg., s.d. (Mus. Par. Collection générale);

-1 déterminée «Clairvillia pauciseta» in coll. Pandellé (Mus. Par., $\mathrm{n}^{\circ} 964$, individu portant le label additionnel de sa détermination rectifiée par mes soins; l'autre $q$ est Eulabidogaster setifacies [Rond.]; les 2 бot sont Labidogyne nitidula [Mg.]).

Matériaux personnels :

- $22 \delta^{\wedge} \sigma^{\star}$ récoltés à Richelieu (Indre et Loire) ;

$-20^{0} \sigma^{0}$ récoltés à Chaveignes (Indre et Loire) ;

- 1 ó élevé de Dicranocephalus agilis à Richelieu (Indre et Loire) (cas P 2578);

- 12 우 récoltées à Richelieu (Indre et Loire).

2) Dionaea aurulans Robineau-Desvoidy.

a) Principaux caractères diagnostiques.

of : Thorax faiblement pruineux, presque totalement noir discalement à l'arrière et peu rayé à l'avant ; scutellum très peu cendré ; 2 ( $(q+)$ ou 3-4 $\left(\delta^{\circ} \delta^{*}\right)$ rangs de sétules orbitales en dehors des macrochètes frontales; orbite à pruinosité irrégulièrement clairsemée sous les sétules. - $\delta \hat{\delta}$ : orbites noirâtres; sétules parafrontales hautes, soies des joues longues; vertex plus étroit (1/3 de l'ail); orbite de la largeur de la bande frontale; macrochètes marginales formant un rang complet à l'arrière des. tergites abdominaux I-II, III, IV et V, mais les médianes sur les tergites I-II et III sensiblement décalées vers l'avant du tergite par rapport aux latérales; tergite I-II noir, les trois suivants marqués d'une pruinosité grise très légère, de sorte qu'à l'œil nu, l'abdomen semble à première vue totalement noir ; forceps médian en forme de sandale, peu recourbé, obtus et sans canelures latérales ni constriction à l'apex. 우: macrochètes abdominales formant, sur les tergites abdominaux I-II et III, un rang complet de marginales dont les médianes sont très sensiblement décalées vers l'avant par rapport aux latérales, et sur le tergite IV, un rang complet inséré presque à la moitié de la longueur de l'urite; abdomen coloré comme dans la $q$ de $D$. aurifrons, mais la pruinosité moins marquée encore. 
b) Matériaux examinés.

Types:

- holotype of de Dionaea aurulans Robineau-Desvoidy, 1830 (Mus. Par., coll. R.D., $\left.\mathrm{n}^{\circ} 302\right)$;

-2 syntypes $\delta$ et 1 allotype $q$ de Dionaea flavisquamis Robineau-Desvoidy, 1863 (Mus. Par., coll. R.D., $\mathrm{n}^{\circ}$ 304). Syn. nov.

Matériaux de diverses collections:

-1 ơ déterminé «Clairvillia aurulans» in Coll. Pandellé (Mus. Par., $\mathrm{n}^{\circ}$ 1823, individu de Gavarnie ou Tarbes, à label blanc; l'autre 3 sous ce nom est aurifrons);

- 1 † déterminée «Clairvillia aurulans》 in Coll. Pandellé (Mus. Par., $\mathrm{n}^{\circ} 1823$ ) ;

$-1 \delta$ et $1 q$ déterminés «Dionaea aurifrons 》 de Marans (Maine-et-Loire), respectivement 21-viII-1921 et 27-vII-1929, J.-Hervé-Bazin leg. (Mus. Par., Coll. J.-H.-Bazin).

- 1 i indéterminée de Délemont (Jura suisse), 7-vIII-1972, A. Freidberg leg. (Tel Aviv Univ., Coll. J.-Kugler).

\section{Travaux cités}

N. B. - Tous les travaux cités ont été con-ulté:-

Bezzi (Mario), 1907. - Tachinidae. — In : Becker (Th.), Bezzi (M.), Kertész (K.) \& Stein (P.): Katalog der paläarktischen Dipteren, Bd 3, Budapest, 1907: 189-597.

Boheman (Carl Heinrich), 1829. - Observationer rörande några Insecters Metamorphose. Vetensk. Acad. Handlingar, f. 1828, Stockholm, 1829: 164-166 (en suédois).

Butler (Edward Albert), 1923. - A biology of the British Hemiptera Heteroptera, 1 vol., $8^{\circ}$, London (H.-F. \& G. Witherby), 1923, I-VIII + 1-682, pl. I-VII, 53 text.-fig., sans $\mathrm{n}^{\circ}$.

CoBben (René H.), 1953. - Bemerkungen zur Lebensweise einiger holländischen Wanzen (Hemiptera-Heteroptera). - Tijdschr. voor Entomol., Deel 96, afl. 3, 1953: 169198, fig. 1-6.

DaLman (Johann Wilhelm), 1828. - Årsberättelse om nyare zoologiska Arbeten och Upptäckter, till Kongl. Vetenskaps-Academien afgifven den 31 mars 1828. Stockholm (K. Vet. Acad.), 1828: I-IV + 1-138 (en suédois).

DupuIs (Claude), 1963. - Essai monographique sur les Phasiinae (Diptères Tachinaires parasites d'Hétéroptères) ( $=$ Contributions à l'étude des Phasiinae cimicophages 25). Mém. Mus. nat. Hist. nat., n.s., Sér. A, Zool., t. 26, 1963 : 1-461, fig. 1-73.

—, 1965. - Contributions à l'étude des Phasiinae cimicophages (Diptères Tachinaires), 30. Notes biogéographiques et taxinomiques sur quatre espèces françaises peu connues. Cahiers des Natur., Bull. N.P., n. s., t. 20 (1964), fasc. 4, 1965: 89-97.

—, 1968. - Contributions à l'étude des Phasiinae cimicophages (Diptera Tachinidae), 35. Recherches taxinomiques et biologiques sur Clairvillia biguttata et les Leucostomatina européens affines. Ibid., n.s., t. 24, fasc. 1, 1968: 1-43, fig. 1-21.

—, 1970. - La rencontre des sexes dans la nature chez les Phasiinae, en particulier chez Gymnosoma intermedia Lw (Diptera, Tachinidae) (= Contributions à l'étude des Phasiinae cimicophages, 36). C.R. Acad. Sc., D, t. 270, n 13, 1970: 1699-1701. 
—, 1972. - Cycle annuel de Gymnosoma intermedia Lw (Diptera Phasiinae) parasite spécifique plurivoltin de l'Hémiptère Pentatomide univoltin Eusarcoris Fabricii Kirk. (= Contribution à l'étude des Phasiinae cimicophages, 37). Bull. Soc. zool. Fr., t. 96 (1971), $\mathrm{n}^{\circ} 3$ (Assembl. générale, Rythmes et cycles biologiques, Rennes 1971), $1972: 353$.

FALLén (Carl Friedrich), 1820. - Diptera Sueciae, vol. II (Fam. 14: Muscides, part 2), $8^{\circ}$, Lund., $1820: 13-24$.

Gulde (Johannes), 1921. - Die Wanzen (Hemiptera Heteroptera) der Umgebung von Frankfurt a.M. und des Mainzer Beckens. Abhandl. senckenb. Naturf. Ges., t. 37, 1921: 329-503, fig. $1-7$.

HALÂSZFY (Eva), 1954. - Magyarország és a környezö területek Brachyplatida és Cydnida fajainak ökológiája és elterjedése. The oecology and distribution of the Brachyplatid and Cydnid species in Hungary and the neighbouring countries. Rovartani Közlemények-Folia ent. Hung., ser. nov., t. 7, $\mathrm{n}^{\circ}$ 8, 1954: 93-132, 1 graph. + 1 papillon erratum (en magyar, résumé anglais).

Herting (Benno), 1957. - Die Raupenfliegen (Tachiniden) Westfalens und des Emslandes. Abhandl. aus. d. Landesmus. f. Naturk. zu Münster in Westf., 19. Jg., 1957: 1-40.

-, 1960. - Biologie der westpaläarktischen Raupenfliegen, Dipt. Tachinidae. Monogr. zur angew. Ent., Beihefte zur Zeitschr. f. angew. Ent., Nr 16, 1960 : 188 pp., fig. 112.

—, 1966. - Beiträge zur Kenntnis der europäischen Raupenfliegen (Dipt. Tachinidae). IX. Stuttgarter Beitr. $z$. Naturk., Nr 149, 1966 : 1-12.

JoRdan (Karl H.C.), 1940. - Die Heteropterenfauna der Oberlausitz und Ostsachsens. Isis Budissina, Bautzen, 14 (1936-40), 1940: 96-156.

KISELEV (A. N.), 1951. - O nekotorykh biotitsheskikh faktorakh ogranitshivaiushtshikh tshislennost' iablonnoi plodozhorki [Carpocapsa pomonella] v Krimu. [Sur quelques facteurs biotiques limitant l'abondance de Carpocapsa pomonella en Crimée]. $2^{a}$ Ekol. Konfer., Kiev 1950, Tez. Dokl., part 3, 1951: 98-103 (en russe).

KORINEK (V. V.), 1940. - K biologii nekotorykh poluzhestkokrylikh nasekomykh sem. Thyreocoridae (Hemiptera-Heteroptera). [Sur la biologie de quelques Hétéroptères de la famille Thyreocoridae (Hemiptera Heteroptera)]. Trudy Khopersk. gos. Zapoviedn., livr. 1, 1940 : 219-244, fig. 1-12 = Utshenie Zapisk. leningr. gos. Univ., 28 (1939), 1940 : 219-244, fig. 1-12 (en russe).

Macquart (Justin), 1835. - Histoire naturelle des Insectes. Diptères. Tome deuxième. $8^{\circ}$, Paris (Libr. de Roret, coll. des Suites à Buffon), 1835:1-703 +2 pp. errata ; pl. XIII-XIX + pp. 1-8.

Meigen (Johann Wilhelm), 1824. - Systematische Beschreibung der bekannten europäischen zweiflügeligen Insekten. 4. Theil. $8^{\circ}$, Hamm, 1824 : XII +428 pp. pl. 33-41.

Michalk (Otto), 1933. - Ueber Wanzenfliegen (Phasiinae, Dipt. Tachinidae). I: Neue Funde. Ent. Zeitschr., t. 47, 1933: 128-130.

—, 1938. - Die Heteropteren der leipziger Tieflandsbucht und den angrenzenden Gebiete. Sitz.-Ber. d. Naturfarsch. Ges. Leipzig, t. 63-64, 1938: 15-188.

MoKrZeCKI (Sigismund A.), 1903. - Thryptocera (Gymnopareia) pomonellae Schnabl \& Mokrz. sp. nov., of \& (Diptera, Muscidae). Rev. russe d'Ent., t. 3, n ${ }^{\circ}$ 3-4, 1903 : 211-214.

Pandellé (Louis), 1894. - Etudes sur les Muscides de France. $2^{\circ}$ partie. Rev. d'Ent., t. 13, 1894: 1-113. [Edition tirée à part : t. 1 : 107-219]. 
PutchKov (Vassilii Georgevitch), 1961. - Shtshitniki [Pentatomoidea]. Fauna Ukraïni, t. 21 (Hemiptera), livraison 1, Kiev (Ak. Nauk. Ukr. RSR, Inst. Zool.), 1961: 1-338, fig. 1-133 (en ukrainien).

Robineau-Desvoidy (Jean-Baptiste), 1830. - Essai sur les Myodaires. Mém. présentés par divers savants (étrangers) à l'Acad. r. des Sc., Sc. math. et phys., t. 2, 1830 : 1-813.

ROYER (Maurice), 1931. - La collection de Diptères de Robineau-Desvoidy retrouvée et transportée au Muséum national d'Histoire naturelle. Bull. Assoc. Natur. Vallée du Loing, Moret, t. 14, 1931: 44-49, 1 portrait.

SCHORR (Hildegard), 1957. - Zur Verhaltensbiologie und Symbiose von Brachypelta aterrima Först. (Cydnidae, Heteroptera). Zeitschr. f. Morph. u. Oekol. d. Tiere, Bd 45, H. 6, 1957 : 561-602, fig. 1-31.

SCHUMACHER (Friedrich), 1916. - Die faunistischen und biologischen Verhältnisse der einheimischen Cydniden. Deutsch. ent. Zeitschr., 1916: 210-213.

SÉGUY (Eugène), 1953. - Diptères du Maroc. Diptera (Encycl. ent., sér. B II), t. 11, 1953 : 77-92.

Singer (Karl), 1952. - Die Wanzen (Hemiptera-Heteroptera) des unteren Maingebietes von Hanau bis Würzburg mit Einschluss des Spessarts. Mitteil. naturwissensch. Mus. Stadt Aschaffenburg, N.F., 5. H., 1952: 3 pp. + 1-128, 1 carte.

Southwood (T. R. E.) \& Leston (Dennis), 1959. - Land and water bugs of the British Isles. 1 vol. $8^{\circ}$, London (Frederick Warne \& $\mathrm{C}^{\circ}$, «The Wayside and Woodland series »), 1959: I-XIII + 1-436, text-fig., 1-153, 63 pl. h.t.

Thomas (D. C.), 1954. - Notes on the biology of some Hemiptera Heteroptera. 1. Introduction and shieldbugs (Pentatomoidea). Entomologist, t. 87, $\mathrm{n}^{\circ} 1089$, febr. 1954, 25-30, illustr.

TownSEND (C. H. Tyler), 1916. - Designations of Muscoid genotypes, with new genera and species. Insec. Inscit. Menstr., t. 4, 1916: 4-12.

Villeneuve (Joseph), 1901. - Observations sur quelques types de Meigen (Dipt.) du Muséum de Paris (suite). Bull. Soc. ent. Fr., 1901: $\mathrm{n}^{\circ}$ 4, 82-85.

-, 1906. - Notes synonymiques sur quelques Muscides. Wien. ent. Zeit., Bd 25, 1906 : $247-$ 248.

-, 1907. - Observations et notes synonymiques concernant quelques Tachinaires types de Pandellé (Dipt.). Ann. Soc. ent. Fr., t. 76, 1907: 379-398.

-, 1931. - Aperçu critique sur le mémoire de P. Stein: «Die verbreitetsten Tachiniden Mitteleuropas ». Konowia, t. 10, 1931: 47-74.

WAGNer (Eduard), 1966. - Wanzen oder Heteropteren. I. Pentatomorpha [sic]. Die Tierwelt Deutschlands..., 54. Teil, Jena (Gustav Fischer), 1966: I-vI $+1-235$, fig. 1-149, 1 carte.

WESTWOOD (John Obadiah), 1840. - Synopsis of the genera of british insects [Appendice à] An introduction to the modern classification of insects. T. 2, London, 1840:1-158 (pagination spéciale). 